Área Abierta. Revista de comunicación

audiovisual y publicitaria

ISSN: 2530-7592 / ISSNe: 1578-8293

\title{
Otro tipo de recompensa narrativa: el concepto de "prolongación temporal" como superación de la serialidad televisiva
}

\author{
Alberto Nahum García Martínez ${ }^{1}$ y Ted Nannicelli²
}

Recibido: 25 de marzo de 2021 / Aceptado: 5 de octubre de 2021

Resumen. Este artículo explora las características de la temporalidad en la televisión; en concreto, las capacidades estéticas propias que la pequeña pantalla tiene a su disposición en virtud de su temporalidad distintiva. Partiendo de una metodología que combina los estudios sobre serialidad que han proliferado tanto en los Television Studies como en la Filosofía de la Estética, este texto apunta, en primer lugar, cómo las nociones existentes de "serialidad" no ofrecen una descripción completamente adecuada del particular carácter temporal de la televisión y de sus capacidades estéticas. Por esta razón, se propone un nuevo concepto: la "prolongación temporal". Esta abarca, a diferencia del concepto de serialidad, tanto la manera específica en la que el medio televisivo emplea la temporalidad, así como los efectos estéticos que este manejo genera en formatos y géneros tan diversos como interminables soap-operas, dramas seriales con arcos argumentales horizontales, procedimentales episódicos, comedias de situación, telediarios, emisiones deportivas, concursos semanales, telerrealidad e, incluso, documentales. Para respaldar nuestra argumentación, el artículo profundiza en tres casos de estudio que apuntalan la validez de nuestra teoría. En primer lugar, se abordan las series de ficción autoconclusivas, de las que se espiga el funcionamiento de la prolongación temporal en los gags recurrentes, en la estructura del monstruo de la semana y en la ruptura que suponen los "episodios especiales". El segundo caso de estudio es la figura del presentador de televisión informativa, donde el concepto de familiaridad establece unas recompensas estéticas privativas gracias al paso del tiempo. El último caso que se examina es el de programas de telerrealidad, con Kitchen Nightmares como botón de muestra, donde las variaciones en las expectativas son esenciales para establecer una comparación fructífera con episodios anteriores.

Palabras clave: Cultura popular; Narrativa; Serialidad; Televisión; Prolongación temporal

\section{[en] Another Kind of Narrative Payoff: The Concept of Temporal Prolongation} As an Improved Alternative to TV Seriality

\begin{abstract}
This paper explores the particularities of television's temporality or, more specifically, the particular aesthetic capacities it has at its disposal in virtue of its distinctive temporality. Drawing from a methodology that combines studies on seriality that have proliferated both in Television Studies and in the Philosophy of Aesthetics, we argue that the extant notions of "seriality" do not offer a full account of television's particular temporal character and the aesthetic capacities it has. For this reason, we dig deeper into a new term: "temporal prolongation". This includes, unlike the concept of seriality, both the specific way in which the television medium uses temporality, as well as the aesthetic effects that this handling generates in formats and genres as diverse as endless soap-operas, serial dramas, episodic procedurals, sitcoms, television newscasts, sports broadcasts, weekly contests, reality TV, and even
\end{abstract}

1 Universidad de Navarra (España).

E-mail: albgarcia@unav.es

ORCID: https://orcid.org/0000-0002-4017-5791

2 University of Queensland (Australia).

E-mail: t.nannicelli@uq.edu.au

ORCID: https://orcid.org/0000-0001-5909-5823 
documentaries. We attempt a definition of "temporal prolongation," distinguishing it from seriality, and we support our claims by working through some assorted examples - of both specific formats and programs - , which shows the validity of our theory. In the first place, we deal with fiction series, where temporal prolongation can work though recurring gags, the monster-of-the-week structure, and the "special episodes." The second case study is the TV news anchor, where the concept of familiarity establishes unique aesthetic rewards thanks to the passage of time. The last case to be examined is that of reality shows, with Kitchen Nightmares as the paradigm, where variations in expectations are essential to institute a fruitful comparison with previous episodes.

Keywords: Popular Culture; Narrative; Seriality; Television; Temporal prolongation

Sumario. 1. Introducción. 2. Marco teórico. 3. Las definiciones de la serialidad. 4. Discusión: hacia una definición de la prolongación temporal. 5. Caso de estudio I: ficción autoconclusiva y sitcoms. 6 . Caso de estudio II: el presentador en programas informativos. 7. Caso de estudio III: la telerrealidad autoconclusiva y Kitchen Nightmares. 8. Conclusión. 9. Bibliografía.

Cómo citar. García Martínez, Alberto Nahum y Nannicelli, Ted (2021). Otro tipo de recompensa narrativa: el concepto de "prolongación temporal" como superación de la serialidad televisiva. Área Abierta. Revista de comunicación audiovisual y publicitaria 21 (3), 349-365, https://dx.doi.org/10.5209/ arab. 75200

\section{Introducción}

Hacia el final de Abed's Uncontrollable Christmas (2x11), un memorable episodio filmado en stop-motion, uno de los personajes de Community (NBC, 2009-15) lanza un golpe bajo a los creadores de Lost (ABC, 2004-10). En su búsqueda del significado de la Navidad, durante un viaje que es tanto literal como figurado, el personaje más obsesionado con la cultura popular de Community, Abed, recibe un regalo: el DVD de la primera temporada de Lost. “¿Ese es el significado de la Navidad?”, le preguntan. "No, es una metáfora", responde Abed, "simboliza la falta de recompensa narrativa". Community es una comedia surrealista y altamente metaficcional. Su malévola referencia a Lost, uno de los relatos seriales por antonomasia, condensa, de manera lúdica, dos rasgos predominantes en el medio televisivo: la dilatación temporal de las historias y su carácter episódico, fragmentario.

La "falta de recompensa narrativa" que Abed le achaca a Lost también simboliza uno de los puntos ciegos de los estudios televisivos contemporáneos: su persistencia en supeditar el estudio de la dilatación temporal al concepto de serialidad, entendida esta como el consumo de varias partes de una historia de manera secuencial, distanciadas unas de otras por días, semanas o años. Antes de continuar, conviene remarcar una disonancia lingüística que puede llevar a error en los lectores hispanohablantes. En inglés se distingue entre serial y series, hasta el punto de que, por ejemplo, los BAFTA británicos tienen premios para ambas categorías. La distinción es clara: los seriales desarrollan, de manera continuada, los arcos argumentales principales a lo largo de toda la temporada, mientras que las series (autoconclusivas) ofrecen capítulos narrativamente autónomos. Sin embargo, en español el término serial parece restringido únicamente a entornos - reales o metafóricos - cercanos a la telenovela.

Sin negar la validez de los estudios que ligan narración y teleserialidad, aquí formulamos un concepto más vasto, que permita abarcar la dimensión temporal de la televisión — esto es, su duración extendida - más allá de los confines teóricos 
de la serialidad. Lo que acuñamos como "prolongación temporal" sirve, pues, para explicar las diferentes maneras de "paso del tiempo" que habilita la especificidad del medio televisivo. Para ello, partiendo de un marco teórico y metodológico que combina los $T V$-Studies con la Filosofía de la Estética repasaremos, en primer lugar, la literatura académica para sintetizar las dos concepciones existentes de serialidad: la simple y la expandida. Después, desarrollaremos las características de nuestra "prolongación temporal", argumentando por qué resulta una demarcación más adecuada para expresar las posibilidades de la dilatación temporal propia de la pequeña pantalla. Por último, ejemplificaremos esta teoría aplicándola a tres casos de estudio deliberadamente dispares que nos permitan demostrar su validez en el ámbito de la ficción televisiva, del entretenimiento y de la información.

\section{Marco teórico}

Nuestro artículo parte de dos preguntas de investigación relacionadas con la cualidad temporal específica del medio televisivo. La capacidad de la televisión para una duración prolongada, ¿qué tiene de específicamente televisiva, si es que existe alguna especificidad? ¿Y qué efectos estéticos únicos, si los hay, son viables en el medio televisivo en virtud de su disposición particular para duraciones prolongadas? Por efectos estéticos simplemente nos referimos a lo que una obra logra en tanto que obra, por ejemplo, si genera risa, suspense, rabia, etcétera. En consecuencia, entendemos que programas tan disímiles como uno de telerrealidad, uno deportivo o una serie de ficción pueden entenderse como obras que engendran determinados efectos estéticos distintivos. En este sentido, Klevan afirma que "la estética no desecha ni degrada el contenido moral, político, emocional, cognitivo o conceptual [de una obra]. [...] No obstante, el engagement será con el valor de su expresión a través de la forma de la obra" (cursiva en el original, 2018: 20).

En el ámbito de los TV-Studies, por supuesto, ya se ha abordado a fondo el asunto de la duración temporal extendida de la televisión. Una de las ideas más estimables de dicha literatura académica es que la reciente ola de dramas televisivos tiene su origen — en cuanto a su estructura narrativa — en formas culturales más antiguas, en concreto las telenovelas y, antes, las radionovelas (Feuer, 1984; Newman y Levine, 2012). A su vez, la estructura narrativa de las radionovelas estaba relacionada con la organización narrativa de otros programas seriales de radio, así como con de las películas seriadas de los albores del cine (The Perils of Pauline [1914], Flash Gordon [1936], Sherlock Holmes [1939-46]). Si seguimos tirando del hilo se constata que el origen de la narrativa serial de larga duración radica en los folletines literarios, esos relatos por entregas victorianos que se popularizaron en paralelo al ascenso de la prensa escrita en las sociedades industriales (Hayward, 1997).

En los últimos años, la literatura académica en el ámbito de los TV-Studies ha seguido prestando atención a las evoluciones y novedades en la práctica de la serialidad en la pequeña pantalla. Ante la generalización del streaming y el visionado al ritmo que cada espectador marque, diversos autores han reflexionado sobre la tensión entre continuidad y disrupción narrativa en este nuevo escenario favorecido por la tecnología. Van Arendonk ha teorizado sobre la vigencia del episodio como medida narrativa esencial en la serialidad televisiva (2019), Buonanno ha abordado, con una precisa perspectiva histórica, el diálogo académico entre la discontinuidad del visio- 
nado semanal tradicional y el novedoso "paradigma Netflix" (2019), y O’Sullivan ha trazado - tomando ejemplos, también, de la novela, el podcast o el cómic - cómo todo relato serial contiene impulsos tanto victorianos como modernistas (2019: 62), de ahí la amplia variedad de posibilidades. Así mismo, el interés actual por la serialidad televisiva se confirma con la reciente publicación del volumen Exploring Seriality on Screen. Audiovisual Narratives in Film and Television. Editado por Ariane Hudelet y Anne Crémiux, el libro aspira a entablar una conversación que permita a los diversos académicos que participan a "analizar a fondo asuntos relacionados con la especificidad del medio, así como con la evolución de la forma audiovisual y sus industrias hoy" (2021: 4).

\section{Las definiciones de la serialidad}

Más allá de esas referencias bibliográficas de los dos últimos años, en la literatura académica reciente se pueden encontrar, grosso modo, dos maneras de entender la serialidad. La primera — que denominaremos "concepción simple"- mantiene que los seriales son relatos contados en fragmentos o partes consecutivas. Por su parte, la segunda teorización de la serialidad — que designaremos "concepción expandida" aborda un fenómeno mucho más amplio: "Lo que parece una simple cuestión de técnica narrativa, cuando se examina más de cerca resulta ser uno de los problemas de la propia modernidad: el problema de renovar algo mediante su duplicación" (Kelleter, 2017: 29).

\subsection{La "concepción simple" y sus límites}

La "concepción simple" de la serialidad es la dominante en los campos de la Teoría Narrativa y los Media Studies. Describe cómo la televisión exprime las posibilidades de una duración expandida: empleando un modo procesional de storytelling que resulta compartido por propuestas cuya disparidad va desde las soap-operas tradicionales hasta los dramas emblemáticos de la "Complex TV" (Mittell, 2015), cuyas historias se desarrollan en episodios y temporadas sucesivas. Carroll sintetiza esta noción de modo causal: "La forma serial, en cualquier medio, descansa sobre el mismo principio: el relato genera ciertas preguntas que la audiencia espera que se respondan, o bien en la presenta entrega, o en una posterior" (1996: 120). La descripción carrolliana de la serialidad se emplaza en el contexto de la soap-opera, un género que aporta a la serialidad una de sus facetas distintivas. Como es lógico, la serialidad concebida de esta manera es un rasgo de prácticamente casi toda la televisión narrativa contemporánea, puesto que la mayoría de la teleficción actual adopta una forma híbrida, que combina elementos de las series autoconclusivas y de los seriales expandidos (Innocenti y Pescatore, 2011: 34).

Hay que remarcar que esta "concepción simple" de la serialidad no es puramente formal, puesto que sus teóricos también hacen hincapié en consideraciones extratextuales. En la definición anterior de Carroll, por ejemplo, la serialidad es una característica formal, sí, pero que induce las expectativas de la audiencia de una manera determinada. Una idea similar reaparece en la definición que Mag Uidihr traza del arte serial. Para consumir adecuadamente - para interactuar con - una obra serial - es decir, para acceder a alguna o a todas las características estéticas relevantes que 
la conforman - "se requiere consumir adecuadamente cada elemento de esa secuencia, siguiendo el orden o la disposición prescrita por la propia obra" (2013: 262).

Esta "concepción simple" de la serialidad es útil, pero tiene limitaciones epistemológicas. Únicamente afronta una de las posibilidades de la televisión para exhibir una duración extendida. La tesis de Mag Uidhir brinda una manera fructífera de distinguir la serialidad en su acepción simple del fenómeno de mayor alcance que denominamos "prolongación temporal", puesto que subraya el componente normativo que conlleva la serialidad. Es decir, existe un modo adecuado de consumir una obra serial: atendiendo a todos los elementos o partes de una obra en la secuencia que prescribe la propia obra. La intuición de Mag Uidhir es correcta y, además, nos permite examinar cómo otras materializaciones de la capacidad televisiva para una duración expandida quedan fuera de esa "concepción simple" de la serialidad. Como veremos en detalle, hay varios formatos (talk-shows, eventos deportivos, gameshows o ciertos realities) en los que el pleno disfrute televisivo no depende de consumir todos los episodios siguiendo la secuencialidad propuesta por la propia obra.

\subsection{La "concepción expandida" y sus excesos}

Si la "concepción simple" de la serialidad se revela demasiado angosta para hacerse cargo de las posibilidades de la duración extendida, ¿qué ocurre con la "concepción expandida"? Aunque esta aproximación teórica resulte más minoritaria, cuenta con una reciente defensa en The Media of Serial Narrative, editado por Frank Kelleter, y donde colaboran estudiosos del relato serial como Jason Mittell, Sean O'Sullivan o Henry Jenkins. En la introducción, Kelleter admite que lo que denominamos "concepción expandida" de la serialidad puede correr el riesgo de estirar el concepto "casi hasta el punto de abarcar la propia noción de cultura popular" (2017: 14). A continuación, apuesta por una noción relativista de la serialidad como razón extratextual - "una práctica de la cultura popular" - en lugar de formalismo narrativo: "un mismo texto puede ser considerado simultáneamente serial y no-serial, dependiendo de la perspectiva desde la que se mire. $\mathrm{O}$, de manera más precisa, dependiendo de la situación histórica en la que se movilizan sus actividades textuales en un sentido u otro" (2017: 15).

Contra Kelleter, hay que recordar que la serialidad dista de ser una cuestión meramente formal o de estar divorciada de las prácticas de recepción. Las características constitutivas de un serial están determinadas por sus creadores, puesto que los seriales son artefactos, es decir, creaciones humanas sobre las que sus creadores ostentan autoridad. Por citar un ejemplo clásico: Grandes esperanzas es una obra serial, aunque los lectores contemporáneos la vean como una novela completa y autónoma. Que ahora pueda leerse de una sentada no varía que Dickens la escribiera como una historia serial, a capítulo por semana. Por regresar a la definición de Mag Uidhir: Dickens prescribió que las partes de Grandes esperanzas fueran leídas en una determinada secuencia y con postergación temporal entre un capítulo y el siguiente.

Kelleter sobreestima el feedback loop que se produce entre productores y consumidores de relatos seriales: "La estética serial no se desarrolla mediante una sucesión cronológica e inequívoca de composiciones terminadas y actualizaciones conscientes [...]. El repetido solapamiento entre publicación y recepción habilita a las audiencias seriales para involucrarse en el progreso narrativo" (2017: 13). Sin duda, el diálogo existe, pero conviene subrayar también cómo los creadores de esos seriales 
dictaminan los límites de la intervención de la audiencia. La implicación de los creadores en el desarrollo de los contenidos seriales es de naturaleza muy diferente a la de la audiencia, dado el privilegio epistémico que los autores ostentan sobre sus trabajos. Por consiguiente, la suposición de Kelleter no parece del todo correcta en cuanto a que un trabajo puede considerarse serial o no-serial dependiendo de la perspectiva del consumidor o de cómo la audiencia se aproxima a ese trabajo después de su creación. Si su concepto busca describir patrones de replicación y variación en la cultura popular, entonces el término para explicar este concepto no debería ser el de serialidad; asimismo, su concepción no aporta luz sobre los rasgos diferenciales de la duración prolongada de la televisión.

Por tanto, ninguna de las dos concepciones consigna de manera plenamente satisfactoria ni la temporalidad particular del medio televisivo ni la potencialidad estética que admite. La "concepción simple" se antoja estrecha, puesto que no es aplicable, por ejemplo, a formatos de no-ficción como el telediario o ciertos programas de entretenimiento de carácter autoconclusivo - al estilo de Kitchen Nightmares o Queer Eye for the Straight Guy - en los que cada semana hay un nuevo "caso" que resolver. La "concepción expandida", por su parte, resulta excesivamente amplia ya que no permite discriminar características diferenciales entre la televisión y otros medios. En consecuencia, al considerar que el término "serialidad" amenaza con confundir las cosas más que clarificarlas, aquí proponemos el sintagma "prolongación temporal" para dar cuenta de cómo la duración extendida permite al medio televisivo unas posibilidades estéticas exclusivas.

\section{Discusión: hacia una definición de la prolongación temporal}

El interés de este artículo en la duración extendida de la televisión sí incluye tanto formas narrativas como no-narrativas, en ficción y no-ficción. Mag Uidhir presenta una noción de serialidad de la que partiremos para desbrozar nuestro concepto. Lo que cataloga como "obra serial imprecisa" (2013: 264) se refiere, en términos amplios, a trabajos que contienen múltiples partes distintas y para las que la adecuada apreciación requiere consumir cada parte, pero no en un orden particular. Esta argumentación en torno a la "obra serial imprecisa" es útil porque tiene la extensión suficiente para referirse a series autoconclusivas en el sentido estándar, específico de la televisión —consideremos, por ejemplo, la caracterización que hace Kozloff de la serie autoconclusiva como "aquellos programas cuyos personajes y escenarios se reciclan, pero cuyas historias concluyen en cada episodio individual" (1992: 90-91) — así como de otros tipos de televisión que pueden entenderse comúnmente como "formatos".

Se antoja una apreciación correcta pero, de nuevo, nuestra hipótesis es que la duración extendida de la televisión tiene implicaciones, también, para nuestro engagement con propuestas no-narrativas y con programas de no-ficción que comparten el mismo tipo de carácter procesional, de temporalidad extendida, que las series autoconclusivas: telediarios, retransmisiones deportivas o formatos de telerrealidad. Justamente, para poder aplicarlo a estos últimos se impone un concepto más amplio que el de "obra serial imprecisa", puesto que, según su teoría, para apreciar correctamente una obra en su totalidad - por ejemplo, una serie autoconclusiva o un programa de telerrealidad - habría que "consumir de manera adecuada cada elemento" de esa serie o programa. Este "cada elemento" se antoja una cláusula demasiado severa. 
En este punto conviene rescatar una concepción de la serialidad que sí nos parece lo suficientemente amplia como para dar cuenta de los diferentes tipos de televisión, cuya adecuada apreciación está modelada por su particular temporalidad y, al mismo tiempo, lo suficientemente ajustada como para explicar algo sobre dichas particularidades. Nos referimos al sentido de la serialidad que Stanley Cavell explora en un artículo donde concibe la serialización en íntima conexión con la idea de fórmulas y formatos: "Lo memorable, lo valioso, lo criticable no es primariamente la obra individual, sino el programa, el formato" (1982: 77). El formato en Cavell ostenta un doble significado: por un lado, connota un formato televisivo en el sentido ordinario - por ejemplo, Informe Semanal o Master Chef_. En esta acepción, predica Cavell, los formatos pueden generarse siguiendo un principio de "serie-episodio" o un principio de "género-miembro" (1982: 79). La televisión, según su visión, "funciona estéticamente siguiendo el principio de serie-episodio" (1982: 79). En ambos casos "cada instancia — cada episodio de una serie o cada miembro de un género - supone una perfecta ejemplificación del formato" (1982: 82). En sentido amplio, sin embargo, lo que Cavell tiene en mente son formatos como las sitcoms o los procedimentales policíacos, donde los episodios no necesitan participar de una trama común, pero sí deben compartir algunos personajes, escenarios e, incluso, estructuras típicas de la trama, como el del crimen por resolver. Cavell tiene la "intuición de que las repeticiones y recurrencias de los seriales guardan una relación significativa con las de las series autoconclusivas, en las que el relato alcanza una clausura clásica cada vez, $y$ realmente esas repeticiones y recurrencias son formas de un requerimiento que el medio televisivo demanda en todos sus formatos" (1982: 81, cursiva nuestra).

La primera parte de la visión cavelliana parece válida: hay algo clave en torno al carácter temporal tanto de series autoconclusivas como de seriales; de ahí nuestra exploración de concepciones más amplias que la "simple". Por otro lado, resulta más problemática su afirmación de que todas estas cuestiones sobre la temporalidad, la procesionalidad, la repetición y la recurrencia atañen a todos los formatos televisivos. Hay programas de una única emisión - retransmisiones musicales en directo o TV-Movies - que no quedan satisfactoriamente analizados en dichos términos, precisamente porque ni demuestran repetición alguna ni hacen uso de ninguna de las capacidades específicas de la televisión para la duración extendida.

Más allá de estos recelos sobre programas de una única emisión, podemos construir nuestra propuesta sobre la teoría de Cavell. En primer lugar, para despejar la ambigüedad del término "serial" y similares, planteamos una expresión diferente: la "prolongación temporal". No es un neologismo empleado a la ligera: lo consideramos necesario para describir el carácter temporal propio de la televisión, así como sus posibilidades estéticas específicas. El sintagma "prolongación temporal" proviene de la teoría musical, donde "lleva consigo la connotación de un desarrollo temporal, aunque en una estructura orgánicamente unificada” (Nannicelli, 2017: 65). Nannicelli lo acuñó para describir un rasgo diferencial de la televisión, un medio que ofrece una variedad de prácticas para crear obras televisivas con límites temporales difusos y expansivos. Introducir este término permite abarcar las diferentes formas que tiene la televisión para la duración extendida, típica de telenovelas como la longeva As the World Turns y el drama premium Game of Thrones, series autoconclusivas como The Simpsons y Law and Order, programas de reportajes como Informe Semanal, magazines deportivos como Estudio Estadio, concursos como Saber y Ganar, y programas de telerrealidad tanto en su vertiente serial — Gran Hermano- 
como autoconclusiva - Pesadilla en la cocina - En todos esos ejemplos - como en los que analizaremos en detalle en la segunda mitad del artículo- la temporalidad prolongada es relevante para los logros estéticos del programa y los espectadores. $\mathrm{O}$, por expresarlo de otra manera, la adecuada apreciación de todas estas retransmisiones requiere entender su cualidad como producto con una temporalidad prolongada.

A este nivel de análisis resulta complicado trascender afirmaciones generales, puesto que la prolongación temporal ostenta significados diferentes para diversos tipos de programas. En algunos casos hay programas que, para que nuestra apreciación sea óptima, requieren consumirlos en orden cronológico debido a la serialización - en su "concepción simple"- que la prolongación temporal crea. En otros casos - como las sitcoms o los programas de deportes - no necesitamos ver los episodios o entregas siguiendo una secuencia concreta, ni, por supuesto, ver todos los episodios de una temporada. Y, sin embargo, la correcta apreciación de esos programas sí que parece depender del reconocimiento de cómo el formato modela sus instancias individuales, así como del nivel de familiaridad con recurrencias anteriores. De un modo más formal podríamos expresarlo así: consumir apropiadamente una obra televisiva requiere atender a cómo su formato permite unas posibilidades estéticas distintivas. Y, por otro lado, ocuparse adecuadamente de cómo un formato de televisión concreto proporciona unas perspectivas estéticas específicas es, a su vez, aprovechar las posibilidades de esa obra en virtud de la capacidad televisiva para la prolongación temporal.

Para aclarar estas reflexiones teóricas, abordaremos tres ejemplos intencionadamente variados, de modo que la heterogeneidad evidencie la validez general de nuestra propuesta. Para ello seguimos la distinción clásica en tres macro-géneros que, partiendo de Barroso y Bustamante, hilvanan Gallardo-Camacho y Lavín (2019: 169): los géneros de ficción — series, TV-Movies, animación—; los géneros informativos, que oscilan desde los telediarios hasta las retransmisiones deportivas, pasando por los magacines de actualidad; $y$, por último, los géneros de entretenimiento, donde se incluye todas las ramas de la telerrealidad, así como concursos y humor.

\section{Caso de estudio I: ficción autoconclusiva y sitcoms}

El primero de los formatos que detallaremos para ilustrar la validez transversal de la "prolongación temporal" es la televisión episódica no-serial, tanto en el terreno dramático - los comúnmente denominados "procedimentales"- como las sitcoms. Como hemos argumentado, la estructura dramática de las teleseries autoconclusivas es similar a la de la telerrealidad de Kitchen Nightmares: a lo Conan Doyle. Ambas parten de un equilibrio que se quiebra al inicio de cada episodio, de modo que la peripecia de los protagonistas implica la restauración del orden: resolver un enigma, encontrar un culpable, curar un paciente o ganar un caso judicial. Cada relato corto del héroe de Baker Street ofrecía el mismo tipo de clausura que un episodio de Law \& Order o CSI Las Vegas. Como resultado, en las series autoconclusivas cada capítulo es relativamente independiente: los personajes principales, escenarios y relaciones dramáticas persisten a lo largo de los episodios, pero las tramas son autónomas, de modo que apenas requieren un visionado continuado o familiaridad previa con la diégesis para comprender el relato por sí mismo. 
Esto no implica que las series autoconclusivas hagan tabla rasa de lo que ocurre en episodios anteriores o que carezcan de memoria interna. El aspecto clave no es la memoria del personaje - que, aunque ligera, existe para asegurar la coherencia interna del texto-, sino la del espectador, que "no necesita memoria de los episodios anteriores para entender y apreciar el episodio actual. Los episodios pueden verse en cualquier orden y pueden saltarse sin poner en riesgo ni la comprensión ni el engagement" (Newman, 2006: 23). Aun así, la ficción autoconclusiva ofrece algunas singularidades que no pueden explicarse únicamente a través del concepto de serialidad, sino mediante la noción de "prolongación temporal". Analizaremos con detalle tres estructuras habituales de la ficción autoconclusiva para explicar nuestro argumento con más profundidad.

\subsection{Los gags recurrentes}

La célebre web "TV Tropes" ofrece una concisa pero generalizada definición de gag recurrente: "Una broma cuyo humor proviene de la repetición, que idealmente se vuelve más divertida cada vez que se repite". Este puede darse dentro de un único episodio - o de una película - . No obstante, la televisión, dada su dilatación, permite una forma específica de lidiar con ellos. El método más habitual es aderezar el relato, de cuando en cuando, con dichas bromas. Una comedia tan popular como Friends (NBC, 19942004), por ejemplo, hizo que su audiencia se acostumbrara a la denominada swearing signal, ese gesto de juntar rápidamente los puños que permitía a los personajes "hacer un corte de mangas sin que se note" ("The One with Joey's New Girlfriend", 4x5). O, a lo largo de sus diez temporadas, la audiencia encuentra diversas versiones del Smelly Cat cantado por Phoebe. En ambos casos la escena puede funcionar y arrancar carcajadas de un espectador ocasional que desconozca el precedente, pero el logro estético resulta más intenso y satisfactorio, gracias a la prolongación temporal, para aquellos espectadores que son seguidores habituales. El disfrute del gag se deriva, en parte, de su existencia dilatada, pero también por las diversas maneras en las que la prolongación temporal puede desencadenarlo y modificarlo.

Precisamente los fans obtienen el máximo rédito de otra posibilidad de la confluencia entre gags y prolongación temporal: la broma recurrente puede erigirse en expectativa de cada episodio, para comprobar la originalidad de la nueva iteración o carcajearse con cómo los guionistas la insertan forzadamente. Así ocurría, por ejemplo, con la manía de Kramer por entrar siempre sin llamar (Seinfeld) o las rocambolescas muertes de Kenny en cada South Park. Pero, también, los running gags pueden funcionar de una manera más "serial", incluso en shows autoconclusivos. How I Met Your Mother (CBS, 2005-14) ofrece un ejemplo en este sentido. En "The Slap" (2x9) Marshall gana una apuesta a Barney. El perdedor elige: "O recibir diez bofetadas seguidas ahora mismo... o cinco bofetadas que pueden distribuirse como el ganador quiera desde ahora hasta la eternidad". Barney opta por la segunda opción y la bofetada se convierte en una espada de Damocles cómica, que pende sobre la sitcom casi hasta su mismísimo finale - el último guantazo sucede en el antepenúltimo episodio-. Como sintetiza Nannicelli,

Después de la recompensa de la primera iteración de una broma, el gag puede estar vinculado a otro, de modo que provoque una recompensa adicional, amplificada. Existe un placer cognitivo particular en percibir cómo esos gags se entretejen a 
lo largo del relato, en "cazar" las escenas en las que parece que la premisa de la broma se está preparando, en anticipar cómo se va a efectuar el remate y, una vez que se ha producido la broma, en reflexionar sobre las decisiones estructurales que prepararon el terreno para el golpe de humor (2017: 81).

Si el placer cognitivo de una broma que se despliega más allá de un episodio de una serie de televisión tiene parte de su atractivo en la propia naturaleza prolongada del relato, también hay otras estructuras que, aunque confinadas en los muros narrativos de un solo episodio, cuentan con la temporalidad dilatada como clave para realzar su disfrute, precisamente, como veremos a continuación, por la tensión temporal entre la parte — el episodio - y el todo - la serie completa-.

\subsection{El monstruo de la semana}

Uno de los tópicos habituales en las narrativas autoconclusivas es el denominado "monstruo de la semana", característico de la trama que se abre y clausura en un mismo episodio. Incluso en los formatos o géneros donde predomina esta estructura repetitiva, la prolongación temporal puede dar cuenta de cómo la exposición recurrente a varias entregas de una serie - el orden secuencial no es necesario para la validez del argumento - estimula en los espectadores un efecto estético distintivo. Sin negar la validez de la mencionada concepción erotética de Carroll, según la cual el relato va generando preguntas que el público espera que sean respondidas, aquí proponemos una visión diferente de las series autoconclusivas. El concepto de "prolongación temporal" añade otra capa de disfrute estético: la familiaridad con una plantilla concreta - una estructura narrativa, una red de relaciones afectivas entre los personajes - mitiga el esfuerzo cognitivo del espectador al mismo tiempo que le permite dedicar más recursos interpretativos a cada nuevo monstruo, crimen o enfermedad de la semana. Es decir, la especificidad de cada nuevo escenario narrativo dialoga con los previos que el espectador ha visto $\mathrm{y}$, en consonancia, este valora el ingenio, rareza, plausibilidad y demás rasgos de la nueva trama. El público no solo demanda resolver la crisis narrativa característica de este tipo de relatos autoconclusivos, sino que también ansía maravillarse ante la originalidad de cada inédita situación propuesta por los creadores.

Un ejemplo ilustrativo: a pesar de contar con un arco serial relevante durante sus primeras dos temporadas - el descubrimiento de la faceta asesina de Hannibal Lecter y su posterior captura-, Hannibal (NBC, 2013-15) sigue una estructura procedimental. Cada semana, el investigador y perfilador del FBI Will Graham tiene que ayudar a resolver un nuevo y siniestro asesinato. Más allá del misterio semanal, parte del atractivo del relato tiene que ver con la perversa inventiva de los crímenes que se cometen. La prolongación temporal hace que el espectador no solo se muestre estimulado por descubrir la siguiente "naturaleza muerta" que Hannibal tiene que ofrecer, sino que también espolea que el espectador vaya configurando internamente un museo de los horrores donde las impactantes imágenes sanguinolentas se imprimen en su memoria, estableciendo un siniestro diálogo estético entre sí.

Esta estructura del "monstruo de la semana" — con sus variantes procedimentales habituales del "caso criminal o legal de la semana" o "la enfermedad de la semana" - cuenta, incluso, con una vertiente en la que la ruptura del patrón estético y narrativo opera con mayor eficacia gracias al concepto de prolongación temporal, 
como analizamos en el tercer y último subapartado de este primer caso de estudio, centrado en la ficción televisiva.

\subsection{El episodio especial}

El último rasgo distintivo que queremos destacar para alumbrar cómo la prolongación temporal opera en la ficción televisiva procedimental es el del "episodio especial", aquellas entregas en las que una ficción televisiva varía notablemente sus rasgos narrativos y estéticos. Por su propia concepción, las posibilidades resultan infinitas, pero es habitual que estas piruetas se concentren en partes concretas de la temporada - el Especial de Navidad, el de Halloween, etc.- o que adopten estructuras narrativas y estéticas concretas - el "Episodio Embotellado", el pastiche, la travesura narratológica, el "clip episode"-. En cualquiera de sus variantes, la noción de "prolongación temporal" resulta esencial para que un episodio especial funcione, puesto que la misma raíz del concepto implica quebrar la regularidad de la fórmula, asombrar al espectador y ensanchar el molde estético-narrativo habitual. En definitiva, el "episodio especial" funciona por su contraste con la estabilidad que la audiencia le concede al relato.

Entre los innumerables ejemplos posibles, por razones de espacio solo podemos escoger uno para iluminar nuestra idea. Tanto los académicos como los críticos televisivos elogian Buffy (The WB/UPN, 1997-2003) como una de las series más relevantes a la hora de expandir su fórmula mediante atrevidas innovaciones estéticas y narrativas. Entre las contorsiones más celebradas de la serie, "Once More, With Feeling" (6x7) proponía un episodio totalmente musical que sorprendió por su audacia formal. Una vez más, la noción de "prolongación temporal" plasma de manera precisa los logros artísticos del episodio, puesto que el espectador ha de estar continuamente adecuando lo extemporáneo de los códigos genéricos del musical al Buffy al que está habituado. Como es evidente, por naturaleza cualquier serie de televisión está siempre jugando con la estabilidad y la novedad. Sin embargo, el "episodio especial" zarandea la estabilidad y, a través del factor sorpresa, la audiencia obtiene una placentera disonancia cognitiva al tratar de dar sentido y familiarizarse con las nuevas reglas del juego.

\section{Caso de estudio II: el presentador en programas informativos}

El segundo caso que exploramos es el de formatos como los noticiarios o la programación deportiva que, de manera destacada, cuentan con un presentador: parece obvio que parte de la atracción que algunos programas despliegan está relacionada con su figura, tanto por su personalidad como por su apariencia. Incluso, en algunos casos, el paso del tiempo y la recurrencia en el visionado de dichos presentadores alberga una suerte de familiaridad: un vínculo emocional o una especie de amistad del espectador para con el presentador. Como explica Bonner,

Vemos a las personalidades televisivas tan a menudo y su imagen pública es tan consistente y coherente que sentimos que las conocemos. $\mathrm{Y}$, al conocerlas, nuestra "entrada" en el programa con el que están relacionadas se hace más fácil y más predecible, y nuestra probabilidad de permanecer por lo que sea que las hace atractivas -su marca distintiva- se realza (2003: 66). 
Pero, ¿existe alguna diferencia esencial entre la familiaridad que el espectador establece con personajes ficcionales como Sito Miñanco — Fariña - o Aída García - Aida - y presentadores reales como Matías Prats, Gran Wyoming o Alberto Chicote, por citar personas y personajes de géneros dispares? En el contexto de la apreciación artística la respuesta parece ser no. Hay evidencia académica que sugiere que la exposición repetida al mismo personaje de una telenovela, un presentador de noticias, un comentador deportivo o el conductor de un programa de telerrealidad puede, bajo las circunstancias adecuadas, promover una fuerte ligadura afectiva entre la audiencia y la persona/personaje en pantalla (véase Klimtt et al, 2011). Este vínculo se construye sobre dos elementos entreverados, relacionados ambos con las nociones de tiempo y relación: la repetición y la memoria.

La repetición parece ser el elemento más relevante en el engagement de los espectadores con los presentadores televisivos. En cada nueva entrega de un programa siempre hay elementos constantes: el escenario, el tono, el horario de emisión o el formato de busto-parlante. En el caso del noticiario de prime-time, por ejemplo, cada noche resulta diferente y nueva porque el propio concepto de noticia demanda informar sobre hechos novedosos. Al mismo tiempo, también existen muchos rasgos estables, familiares o, incluso, idénticos a programas anteriores - el set, el presentador, la careta musical de entrada, la estructura-. Prestar la correcta atención al empleo de la repetición que permite la prolongación temporal nos requiere ejercitar nuestra memoria - unas veces de manera consciente, otras inadvertida - para calibrar que algo no está siendo duplicado. Si no, el espectador se perdería efectos estéticos prominentes que tienen que ver con repeticiones, variaciones y patrones, así como con los placeres estéticos que fomentan. En consecuencia, la memoria de visionados previos sedimenta en el espectador unas iteraciones semánticas precisas: formas de decodificar tal o cual gesto del presentador, inclinaciones políticas ante cuestiones polémicas e, incluso, frases pegadizas y expresiones típicas de bienvenida o despedida.

Esto último merece la pena desarrollarlo, porque esas frases particulares que enuncian los presentadores se convierten en una suerte de firma estampada sobre la propia esencia de la prolongación temporal. Ya sea el "así son las cosas y así se las hemos contado" que popularizó Saénz de Buruaga a finales de los noventa en la televisión española o el mítico y sereno "Buenas noches y buena suerte" con el que Murrow se despedía en los albores de la televisión estadounidense, estas frases pegadizas funcionan como marcadores de prolongación temporal, ergo, de familiaridad. Esa familiaridad también la previó el propio Stanley Cavell cuando apuntaba, refiriéndose al lenguaje no-verbal, cómo Johnny Carson podía "instruir las respuestas de su público con una simple mirada en una dirección [...], un poder que el comediante comparte con el domador de leones" (1982: 88). Por ejemplo, uno de los presentadores de informativos estrella de Fox News, Tucker Carlson, ha acostumbrado a su audiencia a una serie de gestos y sutiles exclamaciones llenas de significado. Sus entrevistas en Tucker Carlson Tonight son una mezcla de antipatía e ironía, filtrada a través de su mirada políticamente conservadora. Como es lógico, este sesgo ideológico genera expectativas en cómo se va a desarrollar cada entrevista. Pero, más allá de esa certeza lógica, el empleo de la pantalla partida anima al espectador a identificar rápidamente el código semántico del presentador, viéndole reaccionar a las respuestas de sus entrevistados: su sonrisa de incredulidad, sus respuestas belicosas o sus sarcásticos "uh" para sugerir duda. Todos estos gestos de 
Carlson inducen al espectador, anunciando una escalada verbal inminente donde el sarcasmo, las interrupciones constantes y un debate acalorado resultan inevitables. En este caso, el espectador es capaz de leer las señales gracias a la prolongación temporal, de modo que puede anticipar un momento climático en el programa o una entrevista memorable.

Lo mismo puede decirse de Bill Maher - un ejemplo del otro extremo del espectro político-, en este caso en un programa más escorado al entretenimiento. El espectador entra en Real Time with Bill Maher consciente de su mentalidad progresista, esperando bromas crueles e ingeniosas a costa de los republicanos. Pero, de nuevo, también existen una serie de expectativas visuales, adquiridas a lo largo de años, gracias a la prolongación temporal. El monólogo semanal de Maher, por ejemplo, añade a las estrategias cómicas genéricas su modo característico de contenerse la risa después de decir una broma con el semblante completamente serio, o su peculiar manera de interactuar con el público - muy progresista - del plató. Un ejemplo extraído de esta interacción puede ilustrar cómo un espectador casual no obtendría el mismo placer estético que uno habitual —no necesariamente serial- En un programa de abril de 2012, Maher hizo un chiste estridente jugando con el juicio a George Zimmerman — quien disparó al adolescente afroamericano Trayvon Martin- y el grupo de música One Direction, formado por chicos irlandeses y británicos. La audiencia en plató, habitualmente muy ruidosa y partidaria del humor irónico de Maher, guardó un incómodo silencio ante su pulla. En consecuencia, el presentador les respondió haciendo una peineta con cada mano mientras gritaba: "¡Oh, que os jodan! Quiero decir, en serio. ¡Tíos, el programa ha empezado! Estamos en la HBO, estamos en California. Podéis sacaros el palo del culo y dejar de ser tan quisquillosos. ¡Solo es una broma! No vamos realmente a pegarles un tiro a esos chicos de One Direction". Para extraer toda la savia de este ejemplo, el espectador debe ser consciente de la tendencia de Maher a la incorrección política, sus conocidos valores izquierdistas clásicos y, por supuesto, la lógica complicidad ideológica con su audiencia en vivo; de cualquier otro modo uno no podría disfrutar $-\mathrm{y}$, probablemente, ni siquiera entender - el súbito arrebato de Maher contra el público en el plató.

Por citar un último ámbito que permita apuntar la validez transversal de nuestra teoría, los deportes son otra área donde es común encontrar presentadores carismáticos donde la prolongación temporal ejerce su capacidad estética. Andrés Montés, por citar un caso icónico, se convirtió en el narrador deportivo español más famoso debido, no solo, a su narración eléctrica, sino también a su peculiar forma de referirse a jugadores y jugadas. Construyó su marca narrando los partidos de la NBA, durante la madrugada, pero ascendió hasta convertirse en el locutor principal de los partidos durante grandes competiciones futbolísticas, el deporte más seguido en España. No es de extrañar que fuera el inventor del vocablo "tiki-taka", el término asociada al estilo de juego de la selección española. La exuberancia de Montes para acuñar apodos para los jugadores - "Mr. Cátering Calderón", "Aerolíneas Jordan”-, aplicar muletillas a ciertas acciones del partido - "pincho de merluza" para los tapones, "ya llegan los sioux" para las remontadas-, inventar un argot propio - "Fútbol con fatatas"-, emplear latiguillos — "jugón", "ratatatatatá"—, insertar frases pegadizas — ¿Dónde están las llaves, Salinas?”, "La vida puede ser maravillosa”- e, incluso, desplegar su habilidad para cantar durante los partidos, convirtieron sus narraciones en una experiencia fuertemente codificada. El espectador que nunca había visto antes una de sus retransmisiones, es poco probable que lo encontrara divertido, sino más 
bien extraño y fuera de lugar. Sin embargo, al estar habituado a su excéntrica personalidad catódica y su jerga inimitable, la audiencia esperaba esas marcas de su estilo, encontrándolas hilarantes y adictivas.

\section{Caso de estudio III: la telerrealidad autoconclusiva y Kitchen Nightmares}

En el ámbito del entretenimiento de no-ficción, la temporalidad es una cualidad esencial para entender la fascinación que ejerce la telerrealidad, en formatos tan dispares como Got Talent, Survivor, Pawn Stars, The Deadliest Catch, Duck Dynasty o Master Chef. En primer lugar, los parecidos entre estos formatos y la serialidad resultan evidentes: la mayoría son episódicos y ofrecen una progresión narrativa en la que el espectador necesita tener en cuenta sucesos previos del relato. Los ejemplos más explícitos en este sentido son los formatos que incluyen un concurso - como Survivor o Big Brother-, puesto que la progresión narrativa está, obviamente, en el corazón de la mecánica del programa: resulta imposible restaurar el orden después de un capítulo cuando cada semana hay concursantes que son expulsados. En menor medida, la prolongación temporal también sirve para explicar los hiatos - el parón que los programas sufren entre una temporada y la siguiente, o entre la primera parte de una temporada y la segunda parte- - Ya sea por la comparación entre una tanda de concursantes de Big Brother entre un año y otro, ya sea por el nuevo exótico escenario donde se desarrolla el último Survivor, o por las peculiaridades y popularidad de los nuevos jurados en cada edición de The Voice o Master Chef, descodificar la tensión semántica existente entre memoria, repetición y variación enriquece la experiencia estética de los programas de entretenimiento — ¿qué aporta dramáticamente el nuevo escenario? La variación en el premio o el añadido de ciertas reglas, ¿qué implica para el impulso dramático del programa? ¿Cómo se parecen los nuevos concursantes a estereotipos de ediciones anteriores? - - En este sentido, no es casualidad que la telerrealidad sea un género muy autoconsciente, repleto de "reuniones", all-stars, "regresos" y varios concursantes cruzando de una rama de una franquicia a otra - Nick Viall puede servir de paradigma en este sentido: ha aparecido en las versiones americanas de The Bachelorette, Bachelor in Paradise y The Bachelor-.

La noción de serialidad no sirve, empero, para dar cuenta de todas las temporalidades distintivas de esta televisión. Por eso, a continuación, expandiremos nuestro argumento escogiendo un famoso formato de telerrealidad no-serial: Kitchen Nightmares (Fox, 2007-14). La franquicia de Gordon Ramsay es el equivalente al procedimental tipo CSI en el ámbito del entretenimiento culinario. Cada episodio renueva personajes y escenarios, pero el relato se clausura en cada nueva entrega, para emprender una nueva aventura en el siguiente episodio. Cada semana hay un restaurante a la deriva que enderezar, propietarios problemáticos con los que lidiar y menús completos que rediseñar. ¿De qué manera el concepto de "prolongación temporal" aquí acuñado permite dar cuenta completa de la temporalidad de Kitchen Nightmares? En primer lugar, vuelven a comparecer algunos rasgos explicados en el epígrafe anterior: Ramsay, como presentador del programa, ha acostumbrado a su audiencia a su insatisfacción cuando visita un local por primera vez, a sus épicos arrebatos de ira e, incluso, a sus tics físicos y verbales - las blasfemias, el chocar esos cinco, los “¿uh? ¿Sí?”- Esas son peculiaridades con las que la audiencia está familiarizada, 
que han forjado un vínculo dramático y narrativo mediante la exposición repetida a la estrella del programa.

Más allá del Ramsay-presentador, Kitchen Nightmares exhibe un patrón estructural donde la prolongación temporal juega un papel decisivo en su sentido más básico: la acumulación narrativa. Como hemos explicado, nuestro concepto está relacionado con la repetición y la memoria. Sin duda, un espectador que ve por primera vez Kitchen Nightmares puede seguir el programa y disfrutarlo, puesto que se trata de un relato autoconclusivo; un espectador leal, por su parte, puede obtener una experiencia estética diferente, más enriquecida, a causa de la prolongación temporal. Mientras que la estructura narrativa resulta consistente a lo largo de cada capítulo - emulando la estructura en cuatro actos de las series dramáticas - la mayor novedad de cada entrega radica en el escenario. Cada nuevo restaurante conlleva un lugar inédito, con su personalidad empresarial y su gente polémica. Por consiguiente, más allá de la extendida relación con el presentador - el propio Ramsay -, el televidente afronta cada nuevo episodio esperando una opción culinaria diferente - pastelería, pizzería, pequeño restaurante tailandés, etcétera—, un nuevo grupo de antagonistas —el propietario caótico, el chef arrogante, el camarero negligente-, y variaciones de los problemas habituales - creativos, financieros, higiénicos, familiares, personales-. Mediante la triangulación de esas tres expectativas, el programa ofrece nuevas historias que la audiencia puede acomodar dentro de los patrones narrativos y dramáticos con los que ya está habituada.

Asimismo, al igual que ocurre con los denominados "episodios especiales" de algunas teleficciones - que hemos examinado en el epígrafe anterior-, Kitchen Nightmares juega ocasionalmente con la sorpresa, al romper alguna de sus expectativas nucleares. Así, por ejemplo, hubo seis ocasiones en las que a Gordon Ramsay sí le gustó la comida de los locales; lo habitual es que la encuentre incomible o asquerosa. O, en Amy's Baking Company (6x16) —un imprevisto también célebre por inédito - el chef Ramsay abandonó por primera y única vez un negocio, aceptando que era imposible ayudarles. Otras variaciones temporales que hay que mencionar - aunque, en este caso, relacionadas estrictamente con el concepto de serialidadson los episodios en los que Ramsay regresa a ver cómo han evolucionado algunos de los locales que él ayudó a reflotar en temporadas anteriores.

\section{Conclusión}

La capacidad para extender un relato durante horas es, como hemos expuesto, uno de los rasgos distintivos de la televisión como medio. Desde ámbitos como la Estética o los TV-Studies, varios académicos han explorado este asunto bajo la etiqueta de la serialidad, tanto en su concepción teórica simple como en la amplia, tal y como sintetizamos en la primera parte de este artículo. Hemos propuesto un término más preciso para dar cuenta de todas las posibilidades temporales que ofrece la pequeña pantalla: la "prolongación temporal". Esta abarca tanto la manera particular en la que el medio televisivo utiliza la temporalidad, así como los efectos estéticos que ese manejo genera. Es decir, se trata de un concepto integrador y exhaustivo, que sirve para exponer los logros estéticos que la temporalidad consigue en formatos y géneros tan diversos como interminables soap-operas, dramas seriales repletos de arcos argumentales horizontales, procedimentales episódicos, comedias de situa- 
ción, telediarios, emisiones deportivas, concursos semanales, telerrealidad e incluso documentales.

Para ilustrar y reforzar nuestra teoría, también hemos examinado varios casos concretos en profundidad, en la segunda parte del artículo. Salvo algún ejemplo español aislado - aunque representativo, como el de Andrés Montes- hemos escogido muestras de la televisión estadounidense, la más popular globalmente. La teoría aquí expuesta es válida para destilar de manera precisa las posibilidades estéticas de la temporalidad televisiva, sin que importe la geografía ni el tipo de programa. Para probar la validez de nuestras afirmaciones, hemos examinado un caso por cada uno de los mega-géneros que tradicionalmente han conformado la parrilla televisiva: la ficción, las noticias y el entretenimiento de no-ficción. Sin embargo, somos conscientes de que algunos de los principales contra-argumentos a nuestra idea pueden provenir de dos elementos - un formato y una práctica - que están ganando fuerza en el ecosistema televisivo de la última década: la serie-antología y el visionado en maratón - binge-watching-.

La antología, cuyo máximo exponente en la actualidad es la célebre Black Mirror, se basa en un formato que vivió su edad dorada en la televisión americana de los años cincuenta. La antología es una colección de episodios en los que no existe la acumulación narrativa; por el contrario, en cada capítulo se produce un reinicio narrativo y diegético total, hasta el punto de que incluso el escenario y el reparto de cada episodio sucesivo se renueva por completo. Este reseteo supone un reto para la idea de prolongación temporal aquí expuesta, ya que habría que escrutar el papel de la temporalidad ante episodios completamente independientes, como los que conforman una antología. Por otro lado, la práctica de maratonear una serie también complica el concepto aquí expuesto, ya que esa manera compulsiva de consumir una historia obvia una de las claves estructurales de la ficción televisiva serial: la constante negociación —-fuente de placer estético - entre el ansia de avance narrativo y la ralentización forzada que suponen los hiatos entre episodios o temporadas. Al igual que la prolongación temporal que hemos estudiado durante estas páginas, estas dos limitaciones han de posponerse a futuras investigaciones en las que poder obtener la recompensa académica ansiada.

\section{Bibliografía}

Bonner, F. (2003). Ordinary Television. Londres: Sage.

Buonanno, M. (2019). "Seriality: Development and Disruption in the Contemporary Medial and Cultural Environment". Critical Studies in Television vol. 14, núm. 2, 187-203. DOI: https://doi.org/10.1177/1749602019834667

Carroll, N. (1996). Theorizing the Moving Image. Cambridge: Cambridge UP.

Cavell, S. (1982). "The Fact of Television". Daedalus, vol. 111, núm. 4, 75-96.

Feuer, J. (1984). "Melodrama, Serial Form and Television Today”. Screen, vol. 25, 4-16.

Gallardo-Camacho, J. \& Lavín, E. (2019). "Los géneros televisivos y la audiencia en diferido en España: la ficción vence a la actualidad”. Estudios sobre el mensaje periodístico, núm. 25, vol. 1, 165-79. DOI: https://doi.org/10.5209/ESMP.63722

Hayward, J. (1997). Consuming Pleasures: Active Audiences and Serial Fictions from Dickens to Soap Opera. Lexington: The University Press of Kentucky. 
Hudelet, A. \& Crémieux, A. (eds.). (2021). Exploring Seriality on Screen: Audiovisual Narratives in Film and Television. Nueva York y Abingdon: Routledge.DOI: https://doi. org/10.4324/9781003044772

Innocenti, V. \& Pescatore, G. (2011). "Los modelos narrativos de la serialidad televisiva”. La balsa de la medusa, vol. 6, 31-50.

Kelleter, F. (2017). "Five Ways of Looking at Popular Seriality", en Frank Kelleter (ed.), Media of Serial Narrative. Columbus: The Ohio State UP, 7-34. DOI: https://doi. org/10.2307/j.ctv10crd8x

Klevan, A. (2018). Aesthetic Evaluation and Film. Manchester: Manchester UP. DOI: https:// doi.org/10.7765/9781526132567.00006

Klimmt, C., Hartmann, T. \& Schramm, H. (2011). "Parasocial Interaction and Relationships", en Jennings Bryant \& Peter Vorderer (eds.), Psychology of Entertainment. New York: Routledge, 291-314.

Kozloff, S. (1992). "Narrative Theory and Television”, en Robert C. Allen (ed.), Channels of Discourse, Reassembled. Londres: Routledge, 67-100.

Mag Uidhir, C. (2013). "How to Frame Serial Art". The Journal of Aesthetics and Art Criticism, vol. 71, núm. 3, 261-65. DOI: https://doi.org/10.1111/jaac.12019

Mittell, J. (2015). Complex TV: The Poetics of Contemporary Television Storytelling. New York: New York UP.

Newman, M. Z. (2006). "From Beats to Arcs: Toward a Poetics of Television Narrative". The Velvet Light Trap, vol. 58, 16-28. DOI: 10.1353/vlt.2006.0033

Newman, M. Z., and Levine, E. (2012). Legitimating Television: Media Convergence and Cultural Status. Nueva York: Routledge.

TV Tropes. (n.d.). "Running Gag”. Recuperado de https://tvtropes.org/pmwiki/pmwiki.php/ Main/RunningGag (fecha de acceso: 18/3/2021)

O’Sullivan, S. (2019). “Six Elements of Serial Narrative”. Narrative vol. 27, núm. 1, 49-64. DOI: $10.1353 /$ nar.2019.0003

Van Arendonk, K. (2019). "Theorizing the Television Episode”. Narrative vol. 27, núm. 1, 65-82. DOI: 10.1353/nar.2019.0004 